\title{
SUBDIVISIÓN DE LA PROPIEDAD AGRICOLA EN UNA REGIÓN DE LA ZONA CENTRAL DE CHILE
}

\author{
Instituto de Economía de la Universidad de Chile
}

* Publicaciones del Instituto de Economía de la Universidad de Chile. N³4. Santiago. 1960. 



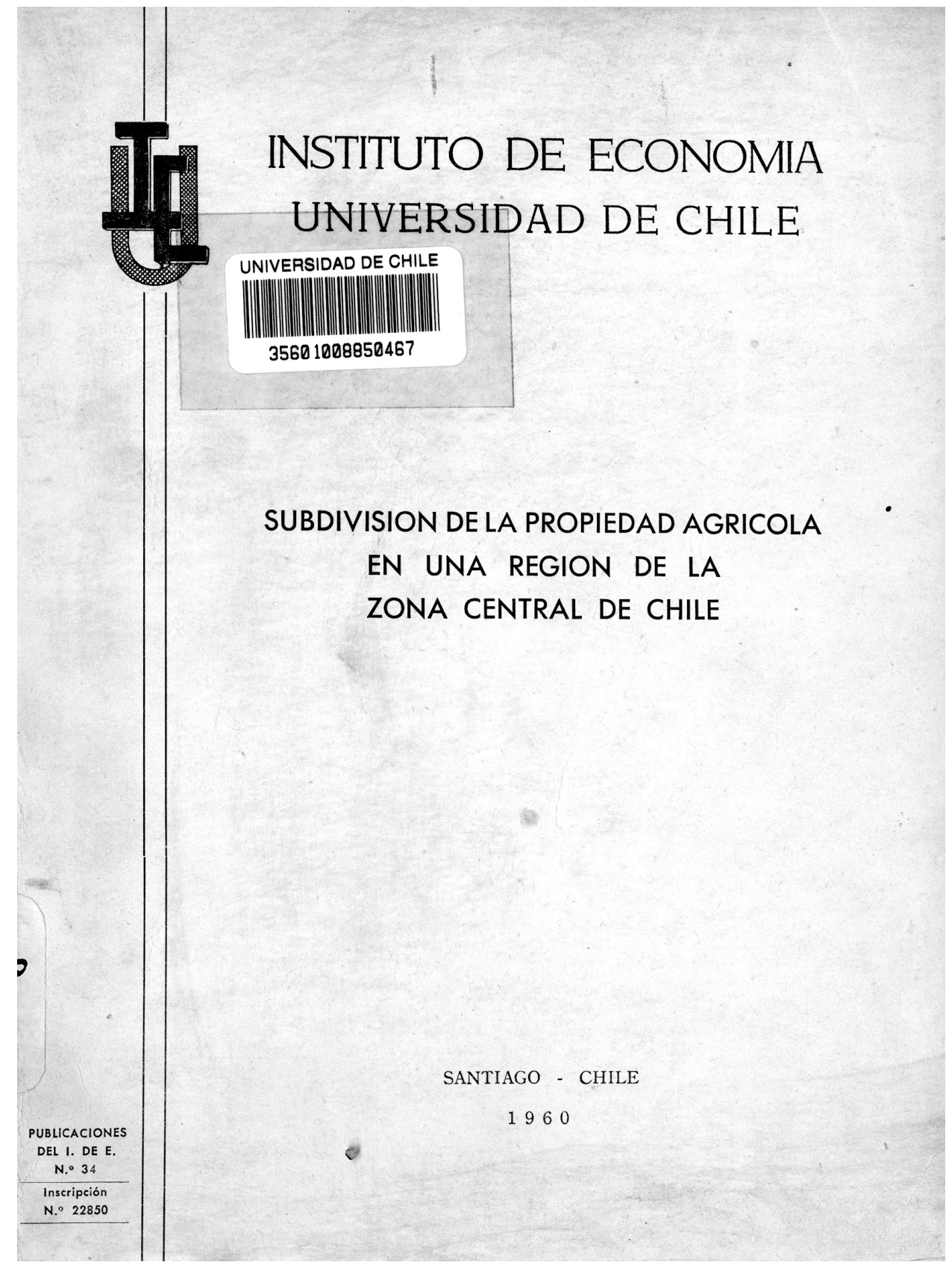




\section{PREFACIO}

E1 presente trabajo tiene por objetivo estudiar algunos antecedentes relacionados con uno de los problemas mas graves de nuestra estructura agraria, a saber, la existencia de un gran numero de propiedades agrícolas muy pequeñas. Muchos autores estân de acuerdo en que la supervivencia y proliferación del minifundio, o parvifundio, provoca situaciones que no se justifican desde el punto de vista económico, y menos desde un ángulo social.

El desajuste basico que causa la propiedad excesivamente pequeña no es difficil de comprender. La relación recurso tierra a hombre es inadecuada para absorber el trabajo de la población que depende de ella y, en consecuencia, la gente debe subsistir en condiciones de subnutricion y de extrema pobreza. En todo caso, ya sea por razones politicas, sociales o económicas, la existencia de la pequeña propiedad, agravada muchas veces por problemas adicionales de fragmentación de los predios, agotamiento del recurso suelos, escasez de agua, y otros, provoca situaciones que deben ser examinadas por la colectividad con el fin de introducir los cambios necesarios.

Una politica estatal destinada a aprovechar integralmente el enorme potencial humano que vive en la pequeña propiedad, acompañada de una consolidación de los predios fragmentados, siempre que el tamaño de las nuevas unidades sea económico, permitiria utilizar la capacidad de trabajo del agricultor y de su familia a la vez que conseguir un nivel de vida adecuado para este sector del agro. Ademas se conseguirian otros objetivos de mucha importancia, como aumento notable de la producción l y mejor utilización de la mano de obra.

Este estudio es uno de los primeros en este pais que trata exclusivamente de este problema. No pretende, sin embargo, agotar el tema ni mucho menos: su contribucion mas importante es la de presentar un panorama del fenómeno estudiado, medir su importancia relativa y analizar algunos aspectos especificos, utilizando cifras obtenidas en un estudio realizado en la zona del rio Rapel, cercana a su confluencia con el estero Alhué.

1/ En algunos países europeos la produccion de los territorios de minifundio se ha duplicado después de la eliminación de éstos. Véase Land Consolidation in Europe. Publication $3 / \mathrm{E}$. International Institute for General Reclamation and Improvement. 
El estudio se divide en cinco capitulos y un apéndice. En el primero se dan a conocer cifras acerca de la importancia del problema de la subdivisión de la tierra en Chile, incluyéndose un estudio sobre la dinămica del proceso.

En el capitulo segundo se presenta la situación existente en la zona del río Rapel aludida, con una descripción de ésta y un estudio histórico del proceso de subdivision en ella.

El capitulo tercero trata de los ajustes economicos que tienen. que ver con la subdivisión excesiva, haciéndose una comparación entre el. minifundio y la propiedad mediana.

En el capitulo cuarto se estudia el origen de la propiedad y de las modalidades de la tenencia de tierras en la zona indicada.

En el quinto se completa la investigación con un analisis del problema de la fragmentación y de la dispersion geográfica de los fragmentos de una explotación.

El apêndice contiene una sintesis de la legislación vigente selacionada en forma directa con el problema de la subdivision de la tierra, una reseña de los resultados de su aplicacion, y breves notas sobre medidas adoptadas en otros paises frente a tal problema.

La investigación estuvo a cargo del ingeniero comercial señor David Alaluf, del Departamento de Investigaciones Económico-Agricolas del Instituto de Economia de la Universidad de Chile.

JOSEPH GRUNWALD

Director

Santiago, septiembre de 1960 


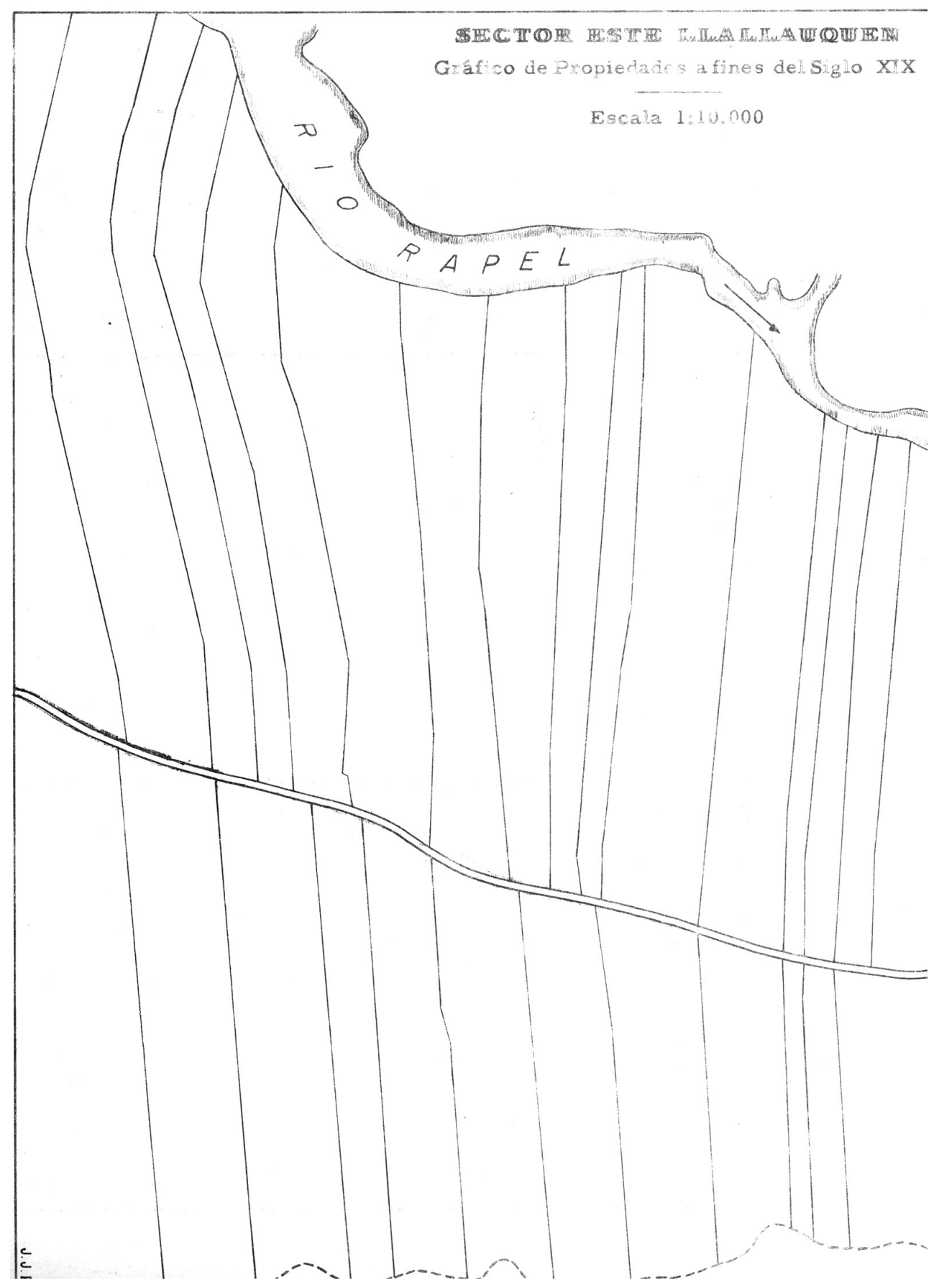




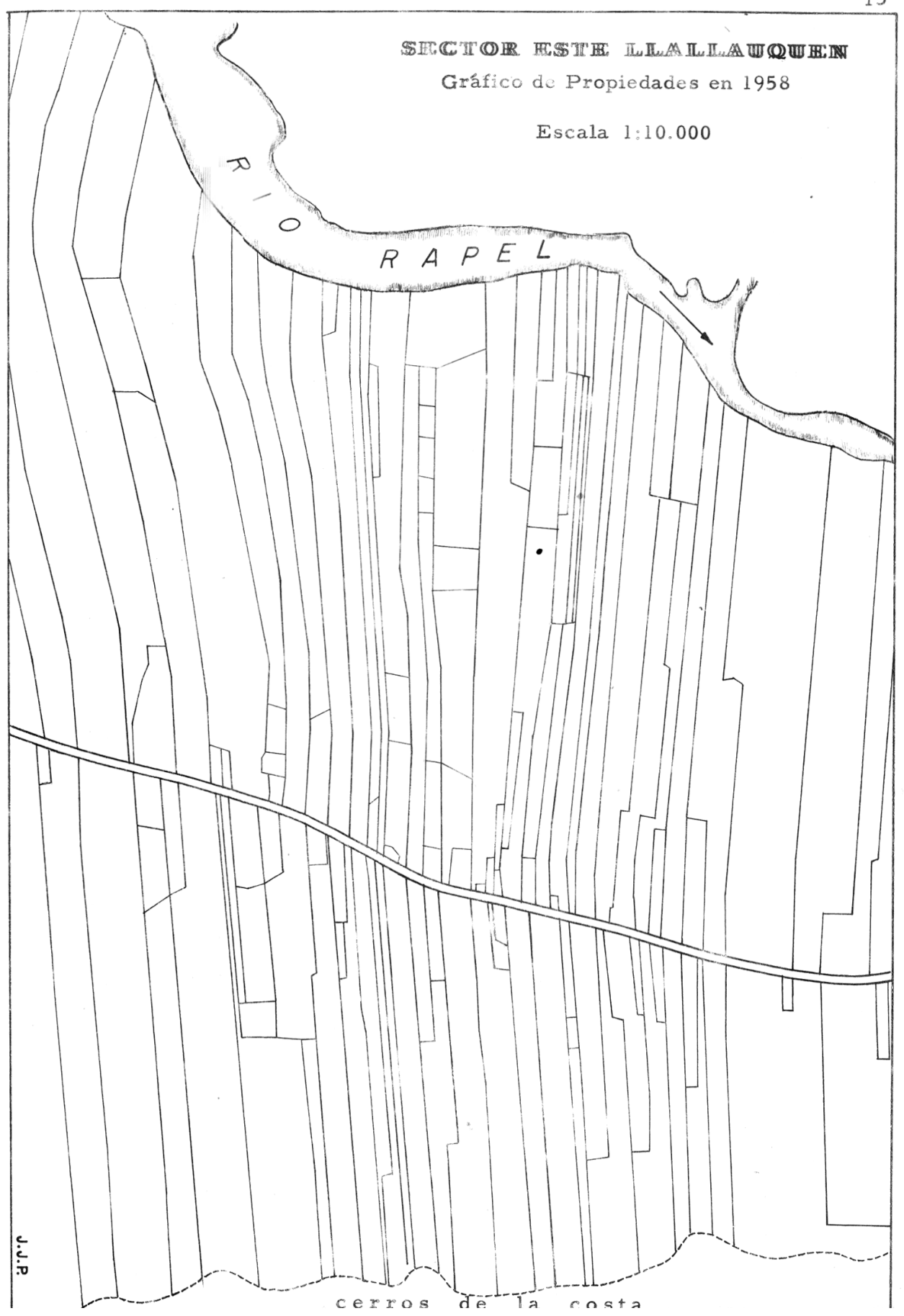




\section{CAPITULO IV}

\section{ORIGEN DE LA SUBDIVISION DE LA TIERRA}

\section{A. Generalidades}

Para entender el proceso de la subdivisión es necesario conocer la forma en que se ha llegado a ella. El cuadro $N^{\circ} 13$ muestra la forma en que fueron adquiridas las 119 propiedades del distrito de Llallauquén elegidas para este estudio.

\section{Cuadro $N^{\circ} 13$}

Modo de Adquisición de 119 Propiedades - en el Distrito de Llallauquén.

\begin{tabular}{|l|c|c|c|}
\hline $\begin{array}{l}\text { Tamaño en } \\
\text { hectáreas }\end{array}$ & $\begin{array}{c}\text { Adquiridas por } \\
\text { herencia }\end{array}$ & $\begin{array}{c}\text { Adquixidas por } \\
\text { compraventa }\end{array}$ & $\begin{array}{c}\text { Namerode } \\
\text { Propiedades }\end{array}$ \\
\hline Menos de 1 Ha. & 27 & 34 & 61 \\
1 a 5 Has. & 18 & 20 & 38 \\
5 a l0 Has. & 4 & 6 & 10 \\
lo Has. y más & 3 & 7 & 10 \\
\hline Totales & 52 & 67 & 119 \\
\hline
\end{tabular}

Fuente: Elaborado por el Instituto de Economía con datos de encuestas propias y realizadas por el Departamento de Economía Agraria del Ministerio de Agricultura y ENDESA.

E1 cuadro revela que son dos las formas en que se ha adquirido el dominio de estas propiedades, a saber, por herencia y por compraventa.

1. Herencia. El 45 por ciento de los predios fue obtenido por herencia lo que en muchos casos significa que la propiedad pasa a manos de varias personas que se la reparten de acuerdo con la voluntad del causante de 1a herencia, o en conformidad a las disposiciones del. Codigo Civil sobre sucesión por causa de muerte cuando no hay testamento. En no pocas ocasiones los herederos no provocan la repartición física del predio y éste permanece como unidad en poder de la sucesión. 
Es común que tratándose de predios relativamente pequeños heredados por personas de bajos ingresos o escaso nivel cultural, ni siquiera se proceda a obtener la posesión efectiva de la herencia.

De las 52 propiedades adquiridas por herencia, 23 están en manos de sucesiones, y de éstas ocho han obtenido la posesion efectiva de ellas.

\section{Cuadro $\mathrm{N}^{\circ} 14$}

Tamaños de Predios y Miembros de Sucesiones que los han heredado

\begin{tabular}{|l|c|c|c|}
\hline $\begin{array}{l}\text { Tamaño en } \\
\text { hectáreas }\end{array}$ & $\begin{array}{c}\text { Numero de } \\
\text { Sucesiones }\end{array}$ & $\begin{array}{c}\text { Numero de } \\
\text { miembros } \\
\text { de la Suc. }\end{array}$ & $\begin{array}{c}\text { Superficie de } \\
\text { los predios } \\
\text { heredados } \\
\text { (hectáreas) }\end{array}$ \\
\hline Menos de 1 Ha & 3 & 13 & 1,12 \\
1 a 5 Has. & 12 & 49 & 26,61 \\
5 a 10 Has. & 5 & 32 & 32,94 \\
10 Has. y más & 3 & 12 & 56,32 \\
\hline Totales & 23 & 106 & 116,99 \\
\hline
\end{tabular}

Fuente: Elaborado por el Instituto de Economía con datos obtenidos en las encuestas citadas anteriormente.

El cuadro anterior muestra que las veintitrés comunidades están compuestas por 106 herederos, cada uno de los cuales es dueño de una parte alicuota de las 117 hectareas heredadas, o sea, que las actuales 23 propiedades pueden transformarse hasta en 106 minifundios de una superficie media de algo más de una hectárea.

En el hecho algunas de estas comunidades se han disuelto extralegalmente, es decir, sus miembros se han repartido la tierra y cada uno administra su parte. En el cuadro $N^{\circ} 15$ se presenta el caso de una comunidad dueña de 5, 9 hectáreas repartidas en doce fragmentos asignados a siete "propietarios" (uno de ellos lo constituyen dos hermanos hijos de una de las herederas). El gráfico que ilustra el caso revela que no siempre los comuneros han conservado sus tierras en un solo paño sino que en dos o más. 


\section{Cuadro $\mathbf{N}^{\circ} 15$}

Repartición de Hecho de la Propiedad de una Sucesión

\begin{tabular}{|c|c|c|}
\hline $\begin{array}{c}\text { Tamaño en } \\
\text { hectáreas }\end{array}$ & $\begin{array}{c}\text { Numero de } \\
\text { fragmentos }\end{array}$ & $\begin{array}{c}\text { Superficie } \\
\text { total } \\
\text { (hectareas) }\end{array}$ \\
\hline 0,200 & 1 & 0,20 \\
0,314 & 4 & 1,30 \\
0,628 & 7 & 4,40 \\
\hline & 12 & 5,90 \\
\hline
\end{tabular}

Fuente: Elaborado por el Instituto de Economía con datos cie las encuestas aludidas.

2. Compraventa. El cuadro $N^{\circ} 13$ muestra que 67 de las 119 propiedades estudiadas fueron adquiridas por compraventa. Del examen de los datos obtenidos en las encuestas se desprende que, en la mayoría de los casos, los compradores llegaron desde regiones donde no habia posibilidad de adquirir pequeñas extensiones de tierras. Un nimero menor de nuevos propietarios está constituído por inquilinos de fundos cercanos a la zona estudiada, que han invertido sus ahorros en adquirir terrenos con el fin de construir en ellos sus viviendas, sin alejarse del sitio de su trabajo principal. $10 /$

$10 /$ Consultados algunos de estos nuevos propietaxios sobre el motivo que tuvieron para elegir la zona estudiada explicaron que en la región donde residian antes no había interés de los dueños de terrenos extensos por desprenderse de partes pequeñas de ellos. En general los grandes propietarios se inclinan a absorber los predios de tamaño reducido que conlindan con sus fundos a fin de evitar los problemas que suelen crear los pequeños empresarios del campo. 


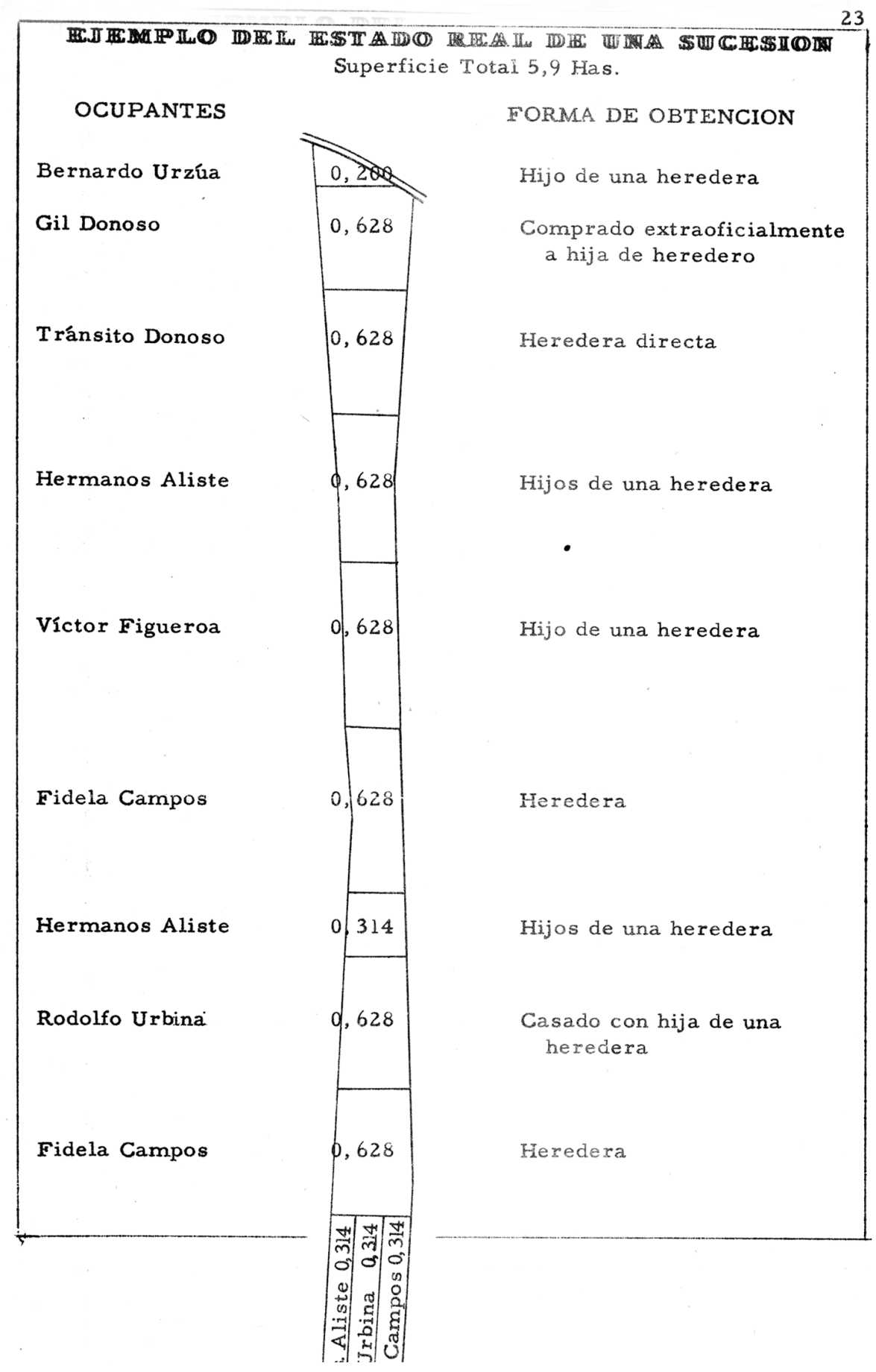


3. Tenencia de la tierra. El cuadro siguiente muestra la forma que adquiexe la tenencia de tiereas en una zona excesivamente subdividida como es la de este estudio.

Cuadro $N^{\circ} 16$

Tenencia de la Tierra

\begin{tabular}{|c|c|c|c|c|c|}
\hline $\begin{array}{c}\text { Tamaño } \\
\text { en } \\
\text { hectáreas }\end{array}$ & $\begin{array}{l}\text { En poder } \\
\text { de sus } \\
\text { dueños }\end{array}$ & $\begin{array}{l}\text { En poder } \\
\text { de dueños } \\
\text { parciales }\end{array}$ & $\begin{array}{l}\text { En poder } \\
\text { de arren- } \\
\text { datarios }\end{array}$ & $\begin{array}{c}\text { Entregada } \\
\text { en } \\
\text { "medias" }\end{array}$ & TOTAL \\
\hline Menos de $1 \mathrm{Ha}$. & 27 & 21 & 2 & 11 & 61 \\
\hline 1 a 5 Has. & 17 & 10 & 20 & 9 & 38 \\
\hline 5 a 10 Has. & 1 & 7 & - & 2 & 10 \\
\hline 10 Has. y más & 7 & - & 3 & - & 10 \\
\hline Total & 52 & 38 & 7 & 22 & 119 \\
\hline
\end{tabular}

Fuente: Elaborado por el Instituto de Economía con datos obtenidos en las encuestas aludidas.

Estas cifras revelan que el 45 por ciento de las propiedades son explotadas por sus dueños y que este porcentaje aumenta a 70 tratándose de predios de diez hectáreas o más. También se presenta el caso de que alguno de los comuneros trabaje las partes de otros comuneros. En esta situación se hallaban 38 de las 119 propiedades estudiadas. Sólo siete predios estaban arrendados a terceros y veintidos habian sido entregados en "medias", sistema que permite al dueño del predio intervenir en la elección de los cultivos 11 \%

IIf "Mediero": el que trabaja tierra ajena, poniendo todo o parte de la semilla, y repartiéndose con el dueño de la tierra la cosecha en proporción preestablecida. 